\title{
Phase rotation-based precoding for spatial modulation systems
}

ISSN 1751-8628

Received on 6th November 2014 Revised on 10th January 2015 Accepted on 22nd February 2015 doi: 10.1049/iet-com.2014.1065 www.ietdl.org

10

\author{
Q1 Ping Yang ${ }^{1}$, Yue Xiao ${ }^{1}$, Bo Zhang ${ }^{2}$, Mohammed El-Hajjar ${ }^{1,2}$, Shaoqian Li ${ }^{1}$, Lajos Hanzo ${ }^{2}$ \\ ${ }^{1}$ National Key Laboratory of Science and Technology on Communications, University of Electronic Science and Technology of China, \\ Sichuan 611731, People's Republic of China \\ ${ }^{2}$ School of Electronics and Computer Science, University of Southampton, Southampton SO17 1BJ, UK \\ d2 $₫$ E-mail: Ih@ecs.soton.ac.uk
}

Abstract: In this study, the authors investigate the benefits of phase-rotation-assisted precoding (PRP) technique in spatial modulation (SM) systems, which are based on maximum free distance $d_{\min }$. First, a closed-form solution of the maximum$d_{\min }$ PRP matrix is derived for the scenario of having two transmit antennas $\left(N_{\mathrm{t}}=2\right)$. Moreover, two numerical methods are

20 proposed for dealing with the case of $N_{t}>2$. The complexity of the proposed algorithms is presented. The authors simulation results show that the proposed PRP algorithms provide beneficial bit error ratio performance improvements compared with both the conventional SM and with the existing adaptive SM.

\section{Introduction}

Spatial modulation (SM) is capable of exploiting the indices of 30 transmit antennas (TAs) as an additional dimension for data transmission besides the traditional amplitude and phase modulations (APMs) [1-5]. Specifically, in this modulation technique, only one of the available TAs is activated during each transmission slot and the information is implicitly conveyed by the index of the activated TA [1]. SM only needs a single-radio-frequency (RF) chain, which is a substantial benefit. As a further advantage, it is capable of relaxing the inter-antenna synchronisation (IAS) specifications, while mitigating the inter-antenna interference (IAI) at the transmitter [6]. Moreover, the single-RF design is capable of employing a low-complexity

40 single-stream maximum-likelihood (ML) detector and offering an increased energy efficiency compared with multipleRF-chain-based schemes. As a result, SM constitutes a promising transmission candidate for multiple-input-multiple-output (MIMO) design [7-9]. It is found that the performances (i.e. bit error ratio 45 (BER) and energy efficiency) of SM-MIMOs are highly dependent on the specific type of the APM scheme [10] used. It has been shown in [11] that phase shift keying (PSK) schemes are good selections for SM systems.

The conventional open-loop SM schemes [11] only offer received

Q3 diversity gains. Hence, there is also a paucity of SM-MIMO

50 literature on how to increase the system's robustness to time-varying channel conditions [12-15]. Specifically, the effects of power imbalance [12, 14], the issues of achieving transmit diversity [13], as well as the impact of cooperation have been researched [15]. However, most of the above-mentioned link 55 adaptive (LA) schemes considered only a special case of SM, namely space shift keying (SSK), which exclusively employs the antenna indices for data modulation. In [16-18], we proposed an adaptive SM (ASM) scheme for improving the achievable BER, while maintaining the target throughput. However, ASM transmits a channel-quality-dependent number of bits per time slot and

60 hence it has a complex detector. More recently, in [19], Maleki et al. first proposed a novel constellation design technique for minimising the symbol error ratio (SER) of the multi-antenna activated SSK scheme, which employs all the TAs to send the same APM symbol. Then, this technique was further developed for the single-RF-aided SSK scheme in [19]. More recently, in [20] the optimal number of bits to be mapped to the TAs and to the APM constellation was investigated under the constraint of a fixed total throughput by the same authors based on a novel tight upper SER bound. However, these LA techniques suffer from symbol-to-symbol transmit power variation, which may cause signal distortion because of the amplifier non-linearity.

To find a high energy-efficiency LA algorithm for SM transmission, the phase-rotation-aided precoding (PRP) technique has also been extended to SM-PSK, in order to increase the minimum Euclidean distance $\left(d_{\min }\right)$ (termed as free distance $\left.(\mathrm{FD})\right)$ of the received constellation points [21]. However, the proposed PRP may only be suitable for the multiple-input-single-output (MISO) systems. Against this background, we introduce a new PRP scheme based on the maximum-FD (max-FD) $d_{\min }$ for SM-PSK systems, which is capable of improving the attainable BER. More important, the symbol-to-symbol transmit power can be constant, which facilities the employment of a high energy-efficiency RF. To retain the benefits of SM, such as its low-complexity single-stream detector and its single-RF chain, we design its PRP matrix to be diagonal. A closed-form solution of the max-FD PRP matrix is derived in the case of two TAs $\left(N_{\mathrm{t}}=2\right)$. To deal with the case of $\left(N_{\mathrm{t}}>2\right)$, we propose two numerical PRP algorithms. We demonstrate that the BER gains achieved by the proposed PRP-aided SM schemes are attractive for high energy-efficiency SM transmission.

Notations: $(\cdot)^{*},(\cdot)^{\mathrm{T}}$ and $(\cdot)^{\mathrm{H}}$ denote conjugate, transpose and Hermitian transpose, respectively. The probability of an event is represented by $P(\cdot)$. Furthermore, $\|\cdot\|$ stands for the Frobenius norm and all logarithms are base of 2 .

\section{System model of minimum Euclidean distance-based PRP for SM-PSK}

\subsection{Transceiver}

The PSK-modulated SM scheme has an attractive BER performance compared with quadrature amplitude modulation (QAM)-modulated SM, as shown in Fig. 1 and it has implementational benefits as well, such as a high-power-amplifier energy efficiency owing to its constant-modulus nature. However, this fixed-mode SM-based scheme only achieves received diversity [6]. For the sake of overcoming this problem, while retaining all the benefits of 

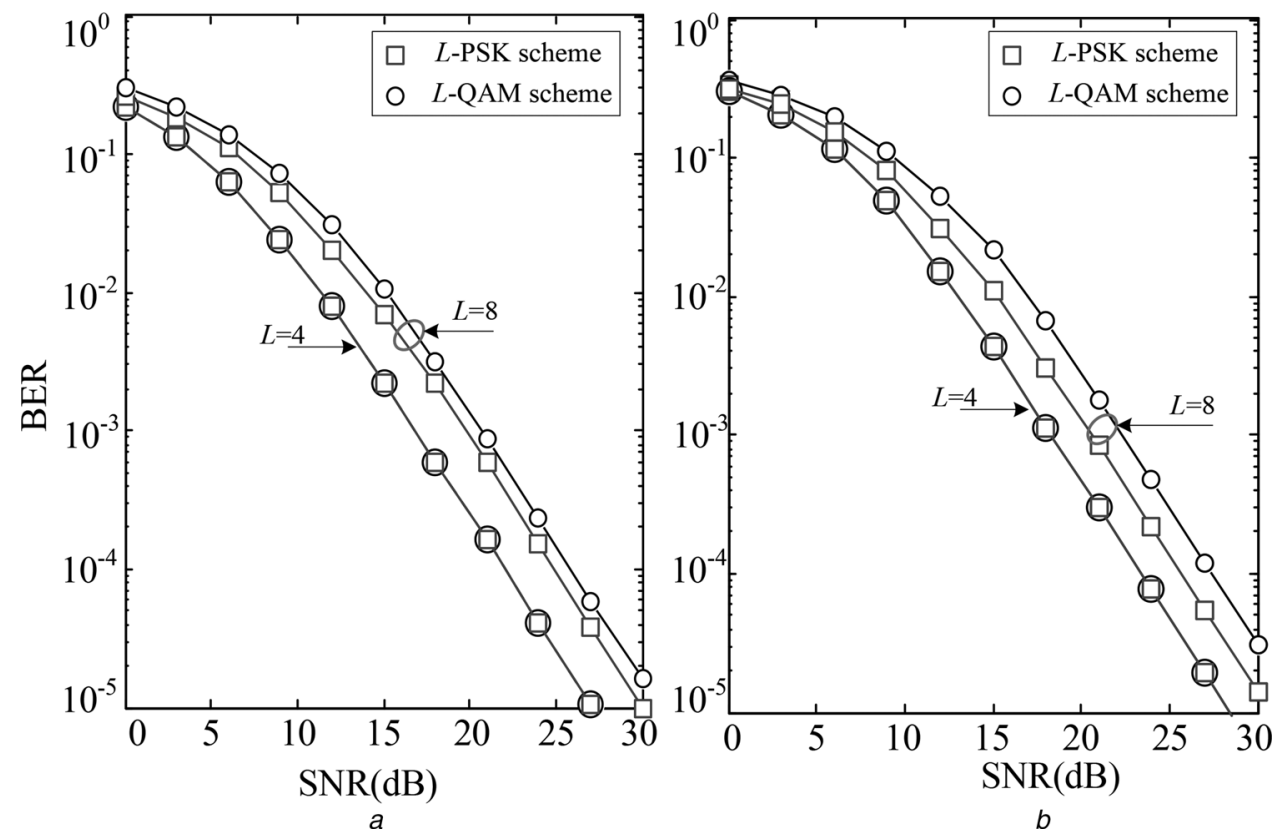

${ }^{15} \mathrm{Q} 4$ Fig. 1 BER comparison of the PSK-modulated SM and the QAM-modulated SM schemes

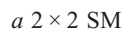

SM-PSK, a PRP-aided SM system is proposed, with the goal of maximising the FD, hence improving the BER performance.

Consider a MIMO system having $N_{\mathrm{t}}$ TAs and $N_{\mathrm{r}}$ RAs. The

Q5 $\left(N_{\mathrm{t}} \times 1\right)$-element transmit symbol vector $\boldsymbol{x}$ is assumed to satisfy $E\left[\boldsymbol{x} \boldsymbol{x}^{\mathrm{H}}\right]=\boldsymbol{I}_{N_{\mathrm{t}}}$, where $\boldsymbol{I}_{N_{\mathrm{t}}}$ denotes an $\left(N_{\mathrm{t}} \times N_{\mathrm{t}}\right)$-element identity matrix. Let $\mathbb{C}$ denote the field of complex numbers. Then, the conventional SM symbol $\boldsymbol{x} \in \mathbb{C}^{N_{\mathrm{t}} \times 1}$ is given by $\boldsymbol{x}=s^{q} \boldsymbol{z}_{q}$, where $s^{q}$ is the complex-valued symbol of the APM scheme. For example, $L$-ary PSK ( $L$-PSK)/QAM is associated with $m_{\mathrm{APM}}=\log$ (L) input bits, whereas $\boldsymbol{z}_{q}\left(1 \leq q \leq N_{\mathrm{t}}\right)$ is selected from the $N_{\mathrm{t}}$-dimensional standard basis vectors (i.e. $z_{1}=\left[\begin{array}{ll}1,0, \ldots, 0 & 0\end{array}\right]^{\mathrm{T}}$ ), according to $\log \left(N_{\mathrm{t}}\right)$ input bits. Hence, a total of $m_{\mathrm{all}}=\log \left(L \cdot N_{\mathrm{t}}\right)$ bits are transmitted in each SM symbol.

Let us express the transmitted PRP-aided SM-PSK symbol as $\overline{\boldsymbol{x}}=\mathrm{e}^{\mathrm{j} \theta_{q}} \boldsymbol{x}=\mathrm{e}^{\mathrm{j} \theta_{q}} S_{l}^{q} \boldsymbol{z}_{q}$, where $\theta_{q}$ is the PRP factor at the $q$ th antenna. At the receiver, the corresponding $\left(N_{\mathrm{r}} \times 1\right)$-element received signal vector is given by

$$
y=H \Theta x+n
$$

where the diagonal matrix $\boldsymbol{\Theta}$ allocates the specific PRP factors to the different TAs, where we have

$$
\boldsymbol{\Theta}=\operatorname{diag}\left\{1, \mathrm{e}^{\mathrm{j} \theta_{1}}, \ldots, \mathrm{e}^{\mathrm{j} \theta_{q}}, \ldots, \mathrm{e}^{\mathrm{j} \theta_{N_{\mathrm{t}}-1}}\right\}
$$

Q6 Given the channel matrix $\boldsymbol{H}$, the PEP between the pair of arbitrary 185 transmit vectors $\boldsymbol{x}_{i}$ and $\boldsymbol{x}_{j}\left(\boldsymbol{x}_{i} \neq \boldsymbol{x}_{j}\right)$ may be estimated as [22]

$$
P\left(\boldsymbol{x}_{i} \rightarrow \boldsymbol{x}_{j} \mid \boldsymbol{H}\right) \simeq \lambda \cdot Q\left(\sqrt{\frac{1}{2 N_{0}} d_{\text {min }}^{2}(\boldsymbol{H}, \boldsymbol{\Theta})}\right)
$$

where $Q(x)=(1 / \sqrt{2 \pi}) \int_{x}^{\infty} \mathrm{e}^{-y^{2} / 2} \mathrm{~d} y$ and $\lambda$ is the number of adjacent constellation points [22], with the $\mathrm{FD} d_{\min }(\boldsymbol{H}, \boldsymbol{\Theta})$ defined as

$$
\begin{aligned}
d_{\min }(\boldsymbol{H}, \boldsymbol{\Theta}) & =\min _{\substack{x_{i}, x_{j} \in \mathbb{X} \\
\boldsymbol{x}_{i} \neq \boldsymbol{x}_{j}}}\left\|\boldsymbol{H} \boldsymbol{\Theta}\left(\boldsymbol{x}_{i}-\boldsymbol{x}_{j}\right)\right\|_{\mathrm{F}} \\
& =\min _{\boldsymbol{e}_{i j} \in \mathbb{E}}\left\|\boldsymbol{H} \boldsymbol{\Theta} \boldsymbol{e}_{i j}\right\|_{\mathrm{F}}
\end{aligned}
$$

where $\mathbb{X}$ is the set of legitimate transmit symbols, while $\boldsymbol{e}_{i j}=\boldsymbol{x}_{i}-\boldsymbol{x}_{j}$, $i \neq j$ denotes the error vector and $\mathbb{E}$ is a set of error vectors.

\subsection{PRP design criterion}

In (4), the conditioned PEP is a monotonically decreasing function of $d_{\min }(\boldsymbol{H}, \boldsymbol{\Theta})$. Hence, the system's BER performance may be improved by maximising the distance $d_{\min }(\boldsymbol{H}, \boldsymbol{\Theta})$ on carefully adapting the transmit parameters. The proposed PRP-aided SM-PSK is designed by appropriately controlling the PRP matrix $\boldsymbol{\Theta}$ for the sake of maximising the FD based on the following cost function

$$
\left\{\begin{array}{c}
\boldsymbol{\Theta}_{\mathrm{opt}}=\underset{\boldsymbol{\Theta}}{\arg \max } d_{\min }(\boldsymbol{H}, \boldsymbol{\Theta}) \\
\text { s. t.: } 0 \leq \theta_{q} \leq 2 \pi, \quad q=1, \ldots, N_{\mathrm{t}}-1
\end{array}\right.
$$

Compared with other existing schemes, the advantages of the proposed PRP scheme are as follows:

(1) In contrast to the traditional transmit precoding conceived for conventional MIMO systems [23, 24], the introduction of single-RF-aided PRP in SM-MIMO does not jeopardise the advantages of SM, such as the avoidance of the IAI and the relaxation of the IAS $[1,6]$.

(2) Compared with the ASM scheme of [16-18] and to the power allocation (PA)-aided SM of [25], the PRP-aided SM only adapts the phases of the SM symbols in order to maximise the FD and hence to improve the attainable BER. As a result, when it is combined with the classic PSK modulation, the moduli of the transmitted signals are constant, which facilitates the employment of a low-cost high-efficiency class-C power amplifier.

\section{Phase rotation-based transmit precoding algorithm}

In this section, we first introduce the conventional PRP algorithm of SM-PSK. Then, we derive a closed-form solution based on the cost function of (5) for binary PSK (BPSK)-modulated $\left(2 \times N_{\mathrm{r}}\right)$-element $\mathrm{SM}$ and then extend this method to the more general $M$-PSK-modulated $\left(2 \times N_{\mathrm{r}}\right)$-element PRP-aided SM scenario. In the case of a large number of TAs and a high modulation order, two 

max-FD solution.

\subsection{Conventional PRP algorithm for MISO channels}

270 In [21], a low-complexity PRP algorithm was proposed for improving the diversity gain of SM in MISO channels, which align the phases of the received constellation points so that they become equi-spaced in $[0,2 \pi]$. To be specific, let the MISO channel vector of $\boldsymbol{h}=\left[h_{1}, \ldots, h_{q}, \ldots, h_{N_{\mathrm{t}}}\right]$, where $h_{q}$ denotes 75 the complex-valued channel coefficient between the $q$ th TA and the receiver, the phase $\theta_{q}$ assigned to the $q$ th antenna is given by

$$
\theta_{q}=\phi_{n}-v_{q}
$$

where $v_{q}$ is the phase of the channel coefficient $h_{q}$ and $\phi_{n}$ is the $n$th angle taken from an equi-spaced angle arrangement within $[0,2 \pi]$, which is given by [21]

$$
\phi_{n}=\frac{2 \pi}{N_{\mathrm{t}} L}(n-1), \quad n \in\left\{1, \ldots, N_{\mathrm{t}}\right\}
$$

The principle of this equally spaced angle arrangement method was detailed, for example, in [21]. As noted in [21], the PRP algorithm of (6) and (7) shapes the received SM symbols so that the angles of the the points are uniformly spaced. This is capable of statistically enhancing the minimum FD and hence improving the achievable BER performance. However, this PRP algorithm is only suitable for the family of MISO channels. To circumvent this problem, we proposed a new PRP algorithm as given in (5). Next, we will drive the solution for this optimisation problem under different SM parameters.

\subsection{Max-FD-aided PRP for BPSK-modulated SM with $N_{t}=2$}

300

In the case of $N_{\mathrm{t}}=2$, the diagonal PRP matrix is formed as $\boldsymbol{\Theta}=\operatorname{diag}\left\{1, \mathrm{e}^{\mathrm{j} \theta_{1}}\right\}$. Hence, we only have to optimise the phase $\theta_{1}$.

For BPSK-modulated SM associated with $N_{\mathrm{t}}=2$, the symbols belong to the set $\{1,-1\}$, and all possible error vectors $\boldsymbol{e}_{i j}=\boldsymbol{x}_{i}$ $\boldsymbol{x}_{j}, i \neq j$ are listed as follows: $\left\{[-2,0]^{\mathrm{T}},[2,0]^{\mathrm{T}},[0,-2]^{\mathrm{T}},[0,2]^{\mathrm{T}}\right.$,

$\left.305[-1,1]^{\mathrm{T}},[-1,-1]^{\mathrm{T}},[1,-1]^{\mathrm{T}},[1,1]^{\mathrm{T}}\right\}$. Since some of the vectors are collinear, the set to be studied is reduced to $\left\{\boldsymbol{e}_{1}, \boldsymbol{e}_{2}, \boldsymbol{e}_{3}, \boldsymbol{e}_{4}\right\}=$ $\left\{[2,0]^{\mathrm{T}},[0,2]^{\mathrm{T}},[1,-1]^{\mathrm{T}},[1,1]^{\mathrm{T}}\right\}$. Given the channel matrix $\boldsymbol{H}=\left[\boldsymbol{h}_{1}, \boldsymbol{h}_{2}\right]$, the constellation point distances at the receiver are given by

On the basis of (8), the optimisation problem of (5) can be simplified to

$$
\begin{aligned}
& \boldsymbol{\Theta}_{\text {opt }}=\underset{\boldsymbol{\Theta}}{\arg \max }\left\{\min \left\{d_{1}, d_{2}, d_{3}, d_{4}\right\}\right\} \\
& \text { s.t. } \quad 0 \leq \theta_{1} \leq 2 \pi
\end{aligned}
$$

To find the optimal PRP solution, we can first obtain the phases to the TAs by finding the intersections of the lines and of the sinusoidal curves in Fig. 2, and then continue by selecting the particular phase resulting in the maximum FD as the final solution. Note that the phase rotation cannot change the values of $d_{1}$ and $d_{2}$, hence only the intersections of the sinusoidal curves have to be

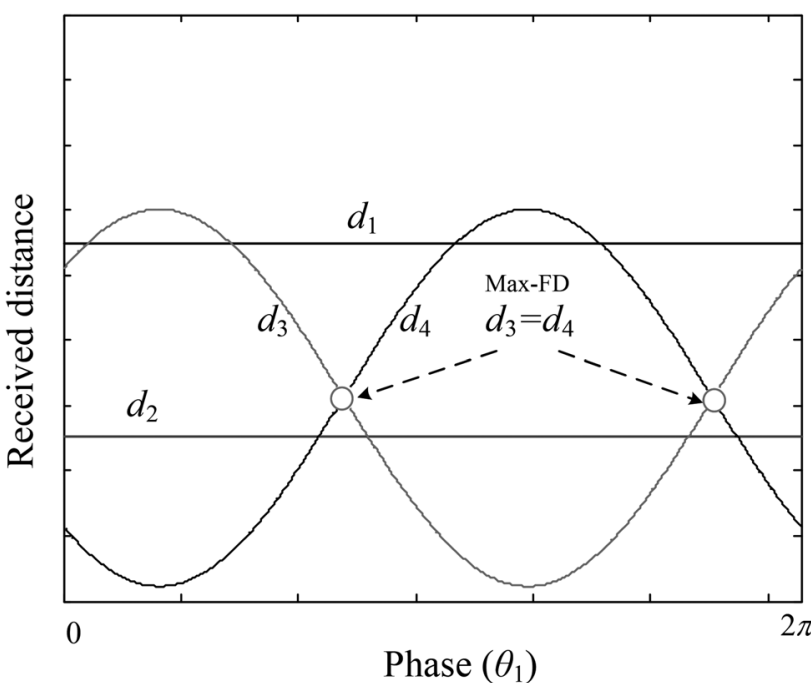

Fig. 2 Max-FD-aided PRP for the BPSK-modulated $(2 \times 1)$-element SM

consider the intersection of $d_{3}=d_{4}$, which satisfies

$$
d_{3}=\left\|\boldsymbol{h}_{1}-\mathrm{e}^{\mathrm{j} \theta_{1}} \boldsymbol{h}_{2}\right\|^{2}=d_{4}=\left\|\boldsymbol{h}_{1}+\mathrm{e}^{\mathrm{j} \theta_{1}} \boldsymbol{h}_{2}\right\|^{2}
$$

leading to

$$
\operatorname{Re}\left\{\boldsymbol{h}_{1}^{\mathrm{H}} \boldsymbol{h}_{2} \mathrm{e}^{\mathrm{j} \theta_{1}}\right\}=0
$$

On introducing the shorthand of $A=2 \cdot \operatorname{Re}\left\{\boldsymbol{h}_{1}^{\mathrm{H}} \boldsymbol{h}_{2}\right\}$ and $B=2 \cdot \operatorname{Im}\left\{\boldsymbol{h}_{1}^{\mathrm{H}} \boldsymbol{h}_{2}\right\}$ for a given channel matrix $\boldsymbol{H}$, (11) can be solved as

$$
\begin{aligned}
& A \cos \theta_{1}-B \sin \theta_{1}=0 \\
& \Leftrightarrow \tan \theta_{1}=\frac{A}{B}
\end{aligned}
$$

Finally, the phase $\theta_{1}$ associated with the intersection of $d_{3}=d_{4}$ in Fig. 2 is given by

$$
\left\{\begin{array}{l}
\theta_{1}=k \pi+\tan ^{-1}\left(\frac{A}{B}\right) \\
k \in Z, 0 \leq \theta_{1} \leq 2 \pi
\end{array}\right.
$$

Note that there may be multiple optimal solutions for (13), as indicated in Fig. 2. Since these solutions result in the same FD $d_{\min }$, we only consider one of them. According to (10) and (13), the distances $d_{3}$ and $d_{4}$ can be expressed as $d_{3}=d_{4}=\left\|\boldsymbol{h}_{1}\right\|^{2}$ $+\left\|\boldsymbol{h}_{2}\right\|^{2}$, then the FD $d_{\min }$ is given by

$$
\begin{aligned}
d_{\min }= & \min \left\{d_{1}, d_{2}, d_{3}, d_{4}\right\} \\
= & \min \left\{4\left\|\boldsymbol{h}_{1}\right\|^{2}, 4\left\|\boldsymbol{h}_{2}\right\|^{2},\left\|\boldsymbol{h}_{1}\right\|^{2}+\left\|\boldsymbol{h}_{2}\right\|^{2}\right\} \\
& =\left\{\begin{array}{l}
\left\|\boldsymbol{h}_{1}\right\|^{2}+\left\|\boldsymbol{h}_{2}\right\|^{2}, \text { if } 3\left\|\boldsymbol{h}_{1}\right\|^{2}>\left\|\boldsymbol{h}_{2}\right\|^{2}>\frac{\left\|\boldsymbol{h}_{1}\right\|^{2}}{3} \\
4\left\|\boldsymbol{h}_{2}\right\|^{2}, \text { if }\left\|\boldsymbol{h}_{2}\right\|^{2} \leq \frac{\left\|\boldsymbol{h}_{1}\right\|^{2}}{3} \\
4\left\|\boldsymbol{h}_{1}\right\|^{2}, \text { if }\left\|\boldsymbol{h}_{2}\right\|^{2} \geq 3\left\|\boldsymbol{h}_{1}\right\|^{2}
\end{array}\right.
\end{aligned}
$$

Remark 2: The conventional PRP algorithm of [21] relying on equi-spaced phases for a $(2 \times 1)$-element SM using BPSK is 
equivalent to the proposed $\max -d_{\min }$-based PRP in terms of maximising the FD.

Proof: Let $\boldsymbol{H}=\left[h_{1}, h_{2}\right]$ be the MIMO channel matrix. For BPSK, the SM system only has four received constellation points corresponding to $h_{1},-h_{1}, h_{2}$ and $-h_{2}$. In the conventional equi-spaced phase-based PRP algorithm, a constant phase difference of $\pi / 2$ is assigned between the adjacent constellation points. Hence, the legitimate received distances are the following three values $4\left\|h_{1}\right\|^{2}, 4\left\|h_{2}\right\|^{2}$, $\left\|h_{1}\right\|^{2}+\left\|h_{2}\right\|^{2}$, which are the same in (14) associated with $N_{\mathrm{r}}=1$. Hence, this method is equivalent to our max-FD-based PRP in this specific SM setup, which will be shown in Fig. 4.

\subsection{Max-FD-aided PRP for L-PSK-modulated SM using $N_{t}=2$}

In this section, we consider the more general case of a $\left(2 \times N_{\mathrm{r}}\right)$-element SM using $L$-PSK modulation. Given the channel matrix $\boldsymbol{H}=\left[\boldsymbol{h}_{1}, \boldsymbol{h}_{2}\right]$, we first classify these received distances into three subsets, as follows

$$
\left\{\begin{aligned}
D_{1}= & \left\{\left\|\boldsymbol{h}_{1}\right\|^{2}\left(s_{l}-s_{\hat{l}}\right), l \neq \hat{l}\right\} \\
D_{2} & =\left\{\left\|\boldsymbol{h}_{2}\right\|^{2}\left(s_{l}-s_{\hat{l}}\right), l \neq \hat{l}\right\} \\
D_{3} & =\left\{\left\|\boldsymbol{h}_{1} s_{l}-\boldsymbol{h}_{2} s_{\hat{l}} \mathrm{e}^{\mathrm{j} \theta_{1}}\right\|^{2}, l, \hat{l} \in\{1, \ldots, L\}\right\}
\end{aligned}\right.
$$

where $s_{l}=\mathrm{e}^{\mathrm{j}(2 l \pi / L)}$ and $s_{\hat{l}}=\mathrm{e}^{\mathrm{j}(2 \hat{l} \pi / L)}$ represent a pair of $L$-PSK symbols. Hence, the optimisation problem of (5) is equivalent to

$$
\begin{aligned}
& \boldsymbol{\Theta}_{\text {opt }}=\underset{\boldsymbol{\Theta}}{\arg \max }\left\{\min \left\{D_{1}, D_{2}, D_{3}\right\}\right\} \\
& \text { s.t. } 0 \leq \theta_{1} \leq 2 \pi
\end{aligned}
$$

Similarly to the BPSK case of Section 3.2, the distances in the sets $D_{1}$ and $D_{2}$ are independent of the phase $\theta_{1}$. As a result, we only have to consider the intersections of the sinusoidal functions in set $D_{3}$. When the phase difference of the PSK symbols $s_{l}=\mathrm{e}^{\mathrm{j}(2 l \pi / L)}$ and $s_{\hat{l}}=\mathrm{e}^{\mathrm{j}(2 \hat{l} \pi / L)}$ is considered, the set $D_{3}$ can be represented by $\hat{D}_{3}$ as

$$
\begin{aligned}
\hat{D}_{3} & =\left\{\left\|\boldsymbol{h}_{1} \mathrm{e}^{\mathrm{j}(2 l \pi / L)}-\boldsymbol{h}_{2} \mathrm{e}^{\mathrm{j}(2 \hat{l} \pi / L)} \mathrm{e}^{\mathrm{j} \theta_{1}}\right\|^{2}, l, \hat{l}=1, \ldots, L\right\} \\
& =\left\{\left\|\boldsymbol{h}_{1} \mathrm{e}^{\mathrm{j}(2(l-\hat{l}) \pi / L)}-\boldsymbol{h}_{2} \mathrm{e}^{\mathrm{j} \theta_{1}}\right\|^{2}, l, \hat{l}=1, \ldots, L\right\} \\
& =\left\{\left\|\boldsymbol{h}_{1} \mathrm{e}^{\mathrm{j}(2 k \pi / L)}-\boldsymbol{h}_{2} \mathrm{e}^{\mathrm{j} \theta_{1}}\right\|^{2}, k=1-L, \ldots, L-1\right\}
\end{aligned}
$$

445

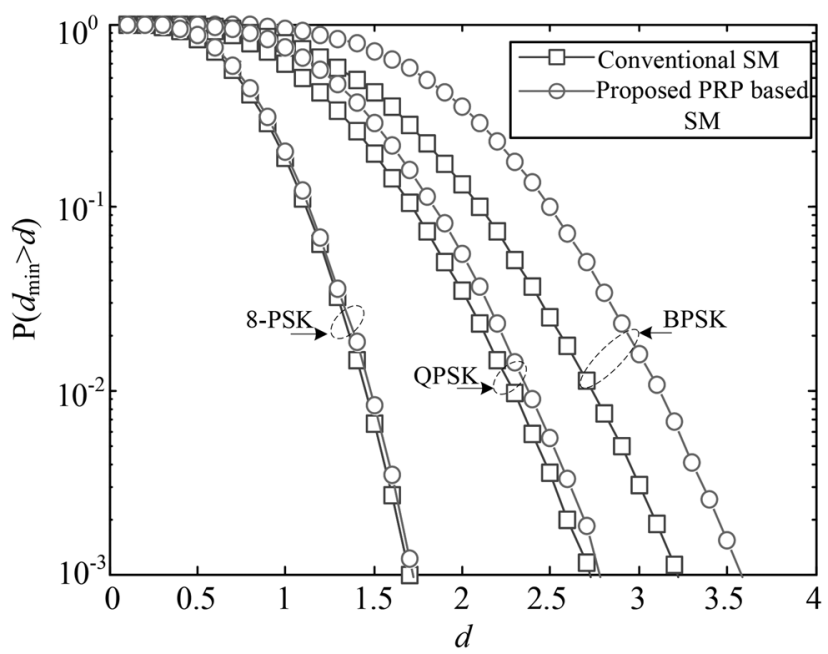

Fig. 3 Distribution of $F D d_{\min }$ of the conventional SM and the proposed optimal max-FD-aided PRP-based SM where the phase differences are $k=l-\hat{l}$. The set $\hat{D}_{3}$ has $(2 L-1)$ elements and we use the phase difference factors $k_{1}$ and $k_{2}$ for representing two elements in the set $\hat{D}_{3}$. If the constraint $2 k_{1} \pi / L=$ $2 k_{2} \pi / L+2 \pi, k_{1}, k_{2} \in(1-L, \ldots, L-1)$ is satisfied, these two elements achieve the same received distance. Hence, we only have to consider one of them. On the basis of this result, the number of elements in the set $\hat{D}_{3}$ is further reduced to $L$. To be specific, only the specific elements of $\hat{D}_{3}$ associated with $k=0, \ldots, L-1$ have to be considered.

On the basis of the above-mentioned process, the problem of (16) is simplified to

$$
\begin{aligned}
& \boldsymbol{\Theta}_{\mathrm{opt}}=\underset{\boldsymbol{\Theta}}{\arg \max }\left\{\min \left\{\hat{D}_{3}\right\}\right\} \\
& \text { s.t. } 0 \leq \theta_{1} \leq 2 \pi
\end{aligned}
$$

Moreover, let $\psi_{1}=2 k_{1} \pi / L$ and $\psi_{2}=2 k_{2} \pi / L$. Then, the interactions between two arbitrary received distances $\left\|\boldsymbol{h}_{1} \mathrm{e}^{\mathrm{j} 2 k_{1} \pi / L}-\boldsymbol{h}_{2} \mathrm{e}^{\mathrm{j} \theta_{1}}\right\|^{2}$ and $\left\|\boldsymbol{h}_{1} \mathrm{e}^{\mathrm{j}\left(2 k_{2} \pi / L\right)}-\boldsymbol{h}_{2} \mathrm{e}^{\mathrm{j} \theta_{1}}\right\|^{2}$ of $\hat{D}_{3}$ in (17) should satisfy

$$
\left\|\boldsymbol{h}_{1} \mathrm{e}^{\mathrm{j} \psi_{1}}-\boldsymbol{h}_{2} \mathrm{e}^{\mathrm{j} \theta_{1}}\right\|^{2}=\left\|\boldsymbol{h}_{1} \mathrm{e}^{\mathrm{j} \psi_{2}}-\boldsymbol{h}_{2} \mathrm{e}^{\mathrm{j} \theta_{1}}\right\|^{2}
$$

When we have $\psi_{1} \neq \psi_{2}$, (19) can be solved as

$$
\begin{aligned}
& A \cos \left(\psi_{1}+\theta_{1}\right)-B \sin \left(\psi_{1}+\theta_{1}\right)=-A \cos \left(\psi_{2}+\theta_{1}\right)+B \sin \left(\psi_{2}+\theta_{1}\right) \\
& \Leftrightarrow \tan \theta_{1}=-\frac{A \cos \left(\psi_{1}\right)-B \sin \left(\psi_{1}\right)-A \cos \left(\psi_{2}\right)+B \sin \left(\psi_{2}\right)}{A \sin \left(\psi_{1}\right)+B \cos \left(\psi_{1}\right)-A \sin \left(\psi_{2}\right)-B \cos \left(\psi_{2}\right)}
\end{aligned}
$$

Since $\psi_{1}$ and $\psi_{2}$ have $L$ different values in (17), we arrive at a total of

$$
\left(\begin{array}{l}
L \\
2
\end{array}\right)=\frac{L^{2}-L}{2}
$$

phase candidates $\theta_{1}$ in (20). As a result, the number of legitimate PRP matrices $\Theta$ becomes $L^{2}-L / 2$. Finally, the FDs of these PRP matrices are generated and then we select the one having the largest FD as our final result.

In Fig. 3, we investigate the FD gain of the proposed PRP-based SM schemes. Specifically, Fig. 3 portrays the complementary cumulative distribution functions of the FD recorded both for conventional SM and for the proposed PRP-aided SM schemes in $(2 \times 2)$-element MIMO channels for different PSK schemes.

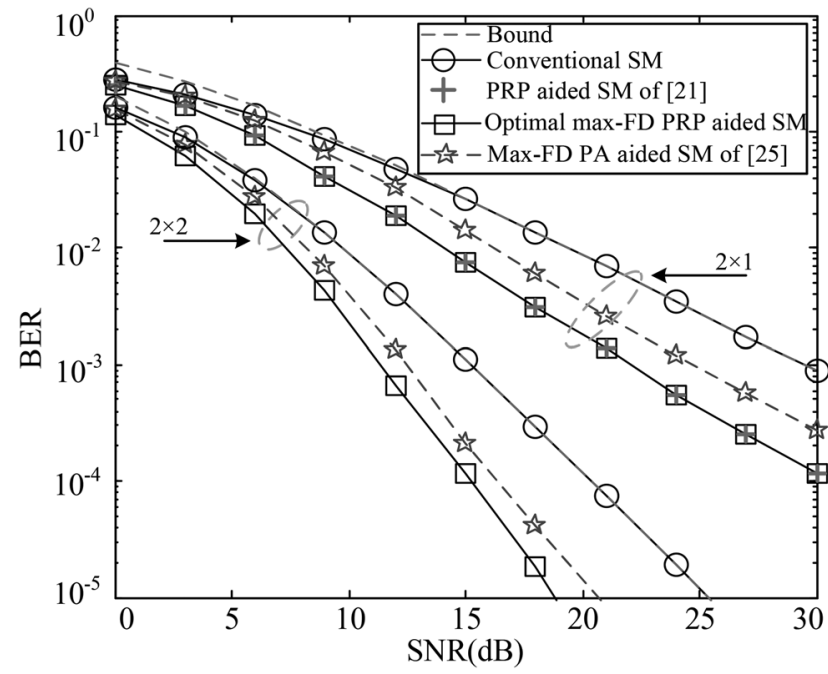

Fig. 4 BER comparison of various SM schemes in $(2 \times 1)$-element MISO and $(2 \times 2)$-element MIMO channels, where BPSK modulation is considered 
Observed in Fig. 3 that the PRP-aided SM schemes are capable of 530 beneficially increasing the FD, hence improving the attainable BER performance according to (3).

\subsection{Exhaustive-based numerical max-FD PRP algorithm}

535 for $N_{t}>2$

Since it is complex to jointly optimise the entire set of phase-rotation values $\theta_{q}, q=1, \ldots, N_{\mathrm{t}}-1$ for the case of $N_{\mathrm{t}}>2$ and for high modulation orders, we simplify this joint optimisation problem by maximising the angular separation of the most likely error-events constituted by the nearest-neighbour TA pair $(m, n)$ associated with the FD. Note that the phase values of the remaining TAs remain unchanged with respect to their initial value. Then, a simplified method of FD calculation may be conceived by exploiting the PRP-SM-specific signal structure.

545 More specifically, if the TA pair $(m, n)$ is associated with the minimum FD according to (4), then problem (5) may be simplified as

$$
\left\{\begin{array}{l}
\boldsymbol{\Theta}_{\text {opt }}=\underset{\boldsymbol{\Theta}}{\arg \max } d_{\min }(\boldsymbol{H}, \boldsymbol{\Theta}) \\
\text { s.t.: } 0 \leq \theta_{m} \leq 2 \pi, m \in\left\{1, \ldots, N_{\mathrm{t}}\right\} \\
\mathrm{e}^{\mathrm{j} \theta_{i}}=1 \text { for all } i \neq m \\
\text { the pair }(m, n) \text { has the minimum FD }
\end{array}\right.
$$

when we consider the nearest-neighbour TAs $m$ and $n$, we only have to rotate one of these antennas, which leads to an improved BER.

More specifically, for a given channel matrix $\boldsymbol{H}$, the $d_{\min }(\boldsymbol{H}, \boldsymbol{\Theta})$ value of the initial PR matrix $\boldsymbol{\Theta}=\boldsymbol{I}_{N_{\mathrm{t}}}$ is calculated as $d_{\min }^{1}$. Then, the indices of the TA pair $(m, n)$ associated with $d_{\min }^{1}$ are obtained. If the value of $m$ is the same as $n$, the distance $d_{\min }^{1}$ is obtained for either one of the pair of TAs, which may be formulated as

$$
d_{\min }^{1}=\left\|\boldsymbol{h}_{m}\right\|_{\mathrm{F}} \bar{d}_{\min }, \quad m \in\left\{1, \ldots, N_{\mathrm{t}}\right\}
$$

565

where $\boldsymbol{h}_{q}$ is the $q$ th column of $\boldsymbol{H}$ and $\bar{d}_{\min }$ is the minimum distance of the classic signal constellation according to the modulation order. For example, we have $\bar{d}_{\text {min }}=2$ for BPSK and $\bar{d}_{\text {min }}=2 / \sqrt{2}$ for quadrature PSK (QPSK) [10]. In this case, no PRP is adopted because it cannot increase the FD of (4). On the other hand, if the 570 values of $m$ and $n$ are not the same, the value of $d_{\min }^{1}$ is computed from different TAs. To increase the spatial separation between these worst-case TAs having the minimum FD, PRP gradually rotates the phase of the $m$ th TA by $\Delta \theta$, whereas the remaining TAs may retain their original phase values. The specific choice of $\Delta \theta$ is

575 flexible and a lower value of $\Delta \theta$ may achieve a better performance at the cost of a higher computational complexity. In practice, the choice of $\Delta \theta$ should strike a tradeoff between the performance attained and the complexity imposed. In this paper, $\Delta \theta$ is chosen as $\Delta \theta=2 \pi / N$ using $N=20$. As a result, the corresponding PRP 580

$$
\boldsymbol{\Theta}_{k}=\operatorname{diag}\left\{1, \ldots, \mathrm{e}^{\mathrm{j}(k \Delta \theta)}, \ldots, 1\right\}, k=0,1, \ldots, N
$$

Table 1 Proposed iterative PRP algorithm for $N_{t}>2$

Initialise $\Theta=\operatorname{diag}\left\{1, \mathrm{e}^{\mathrm{j} \theta_{1}}, \ldots, \mathrm{e}^{\mathrm{j} \theta_{N_{\mathrm{t}}-1}}\right\}=I_{N_{\mathrm{t}}}, b=1$.

Calculate the FD $d_{\min }(\Theta)$ of the PRP matrix and find the indices of the TA pair $(m, n)$ that achieved this FD. If $m=n, \boldsymbol{\Theta}_{\mathrm{opt}}=\boldsymbol{\Theta}$; else go to Step 3.

590 Find the PRP solutions $\Theta_{\text {all }}$ for this TA pair by using (18)-(20) and select the optimal PRP matrix given by $\Theta_{\text {opt }}=\arg \max \left(d_{\min }\left\{\Theta_{\text {all }}\right)\right\}$.

While $d_{\min }\left(\Theta_{\mathrm{opt}}\right)<d_{\min }(\Theta)$, let $b=b+1$ and find the TA pair with the $b$ th minimum distance, go to Step 3 . which yields the optimal PR matrix of

$$
\boldsymbol{\Theta}_{\text {opt }}=\left\{\begin{array}{cc}
\overline{\boldsymbol{I}}_{N_{\mathrm{t}}}, & \text { if } m=n \\
\underset{\boldsymbol{\Theta}=\boldsymbol{\Theta}_{k}, k=1, \ldots, N}{\arg \max } d_{\min }(\boldsymbol{H}, \boldsymbol{\Theta}), & \text { if } m \neq n
\end{array}\right.
$$

Since the FD $d_{\min }(\boldsymbol{H}, \boldsymbol{\Theta})$ is increased by the PRP algorithm, the proposed scheme provides a considerable system performance improvement over the conventional non-precoded SM-PSK.

\subsection{Low-complexity iterative PRP algorithm for $N_{t}>2$}

In Section 3.4, we propose a numerical approach based on exhaustive search, which may have an excessive complexity, when the number of legitimate PRP matrices $N$ is large. Furthermore, it is quite a challenge to decide about the optimal value $N$ for a specific MIMO setup. To solve this problem, based on the optimal solution for provided $N_{\mathrm{t}}=2$ in Section 3.3, we propose a low-complexity iterative PRP algorithm for $N_{\mathrm{t}}>2$, which is summarised in Table 1 . To be specific, similar to the exhaustive-search-based method, we only focus on the specific TA pair $(m, n)$ associated with the FD and use the result of the optimal design for the case of $N_{\mathrm{t}}=2$, whereas the phases of all other TAs remain unchanged. Note that any change in the phase parameters on the TA pair $(m, n)$ may also affect the distances from other TAs. Hence, in order to guarantee that the FD is always increased, we select the specific PRP solution associated with the highest FD by considering all possible TAs. Moreover, if the FD is not increased in an iteration, we continue with the particular TA pair that has the second smallest distance among all TA pairs. In the algorithm of Table 1, we have considered all TA pair candidates up to the $b$ th minimum distance for finding the final PRP result.

Note that for conciseness, we denote the proposed exhaustive-search-based max-FD PRP algorithm as 'the proposed PRP Algorithm 1' and the proposed low-complexity iterative PRP algorithm as 'the proposed PRP Algorithm 2'.

\section{Complexity analysis}

\subsection{Computational complexity-reduction methods}

Since we have a closed-form solution for the conventional PRP as well as for the optimal max-FD PRP methods, their computational complexity is low. However, for a higher number of TAs, the near-optimal max-FD-based algorithm in Sections 3.4 and 3.5 is preferred.

The computational complexity of the numerical PRP algorithm is dominated by that of the phase rotation required for increasing the FD $d_{\min }(\boldsymbol{H}, \boldsymbol{\Theta})$ for each legitimate PRP matrix candidate. To reduce the complexity imposed, below we proposed a range of efficient methods for its practical implementation.

4.1.1 Shrinking the space of error vectors: It is worth noting that the collinear error vectors $\boldsymbol{e}_{i j}=\boldsymbol{x}_{i}-\boldsymbol{x}_{j}, i \neq j$ generate the same FD $d_{\min }(\boldsymbol{H}, \boldsymbol{\Theta})$ in (4). Hence, although the cardinality of the set $\mathbb{E}$ may be large in (4), only a single representative of those collinear error vectors has to be considered. Let $\mathbb{E}^{\prime}$ denote the reduced set of error vectors, where the redundant collinear elements have been eliminated. For a BPSK-modulated SM-based system associated with $N_{\mathrm{t}}=2,4,8$, the entries of the error vectors $\boldsymbol{e}_{i j}$ lie in the set $\{0, \pm 1, \pm 2\}$ and the cardinality of $\mathbb{E}^{\prime}$ is 4,16 and 64 , respectively, which is half of the total number of error vectors. For a higher-order modulation scheme, this reduction of the search space becomes more substantial.

4.1.2 Complexity-reduction by exploiting the PRP-aided $S M-P S K$-specific signal structure: To further reduce the complexity of calculating $d_{\min }\left(\begin{array}{ll}\boldsymbol{H} & \boldsymbol{\Theta}\end{array}\right)$, the PRP-aided SM-PSK-specific signal structure can be exploited. More specifically, let $\boldsymbol{x}_{i}=s_{l}^{i} \boldsymbol{e}_{i}$ and $\boldsymbol{x}_{j}=s_{k}^{j} \boldsymbol{e}_{j}$ denote two distinct transmit 
symbols, and $s^{i}$ and $s_{k}^{j}$ denote the $l$ th and $k$ th constellation points transmitted by the $i$ th and $j$ th antennas, respectively. Then the FD is represented by (25)$$
d_{\min }(\boldsymbol{H}, \boldsymbol{\Theta})
$$$$
=\min _{s_{l}^{j}, s_{k}^{j} \in S}\left\|\overline{\boldsymbol{H}}\left(s_{l}^{i} \boldsymbol{e}_{i}-s_{k}^{j} \boldsymbol{e}_{j}\right)\right\|_{\mathrm{F}}
$$$$
\left.=\min _{s_{l}^{j}, s_{k}^{j} \in S} \| \overline{\boldsymbol{h}}_{i} s_{l}^{i}-\overline{\boldsymbol{h}}_{j} s_{k}^{j}\right) \|_{\mathrm{F}}
$$$$
=\min _{s_{j}^{j}, s_{k}^{j} \in S} \sqrt{\left(\overline{\boldsymbol{h}}_{i} s_{l}^{i}-\overline{\boldsymbol{h}}_{j} s_{k}^{j}\right)^{\mathrm{H}}\left(\overline{\boldsymbol{h}}_{i} s_{l}^{i}-\overline{\boldsymbol{h}}_{j} s_{k}^{j}\right)}
$$

$$
=\min _{s_{l}^{j}, s_{k}^{j} \in S} \sqrt{\left|s_{l}^{i}\right|^{2} \overline{\boldsymbol{h}}_{i}^{\mathrm{H}} \overline{\boldsymbol{h}}_{i}+\left|s_{k}^{j}\right|^{2} \overline{\boldsymbol{h}}_{j}^{\mathrm{H}} \overline{\boldsymbol{h}}_{j}-\left(s_{l}^{i}\right)^{*} s_{k}^{j} \overline{\boldsymbol{h}}_{i}^{\mathrm{H}} \overline{\boldsymbol{h}}_{j}-s_{l}^{i}\left(s_{k}^{j}\right)^{*} \overline{\boldsymbol{h}}_{i} \overline{\boldsymbol{h}}_{j}^{\mathrm{H}}}
$$

where $\overline{\boldsymbol{H}}=\boldsymbol{H} \boldsymbol{\Theta}$ is the effective channel matrix and $\overline{\boldsymbol{h}}_{q}$ is the $q$ th column of $\overline{\boldsymbol{H}}$. Moreover, let us define $\phi=\angle\left(\left(s_{l}^{i}\right)^{*} s_{k}^{j}\right)=-\left(s_{l}^{i}\left(s_{k}^{j}\right)^{*}\right)$, whose legitimate values are limited to

$$
\phi=k \cdot 2 \pi / N(k=0, \ldots, N-1)
$$

Hence, by exploiting that $\left|s_{l}^{i}\right|=\left|s_{k}^{j}\right|=1$ for PSK-modulated symbols, (25) may be rewritten as

$$
d_{\min }(\boldsymbol{H}, \boldsymbol{\Theta})=\min _{s_{l}^{j}, s_{k}^{j} \in S} \sqrt{\left|\boldsymbol{h}_{i}\right|^{2}+\left|\boldsymbol{h}_{j}\right|^{2}-2 \operatorname{Re}\left\{\mathrm{e}^{\mathrm{j}\left(\phi-\theta_{i}+\theta_{j}\right)} \boldsymbol{h}_{i}^{\mathrm{H}} \boldsymbol{h}_{j}\right\}}
$$

where $\left|\boldsymbol{h}_{i}\right|^{2},\left|\boldsymbol{h}_{j}\right|^{2}$ and $\boldsymbol{h}_{i}^{\mathrm{H}} \boldsymbol{h}_{j}$ are the elements of the matrix $\boldsymbol{H}^{\mathrm{H}} \boldsymbol{H}$. For a fixed TA pair $(i, j)$ in (27), we only have to consider the legitimate phase $\phi$. Hence, the corresponding complexity of the PRP algorithm is low.

\subsection{Complexity of the proposed PRP algorithms}

On the basis of the above-mentioned complexity-reduction methods,

Table 2 shows the complexity comparison of various ASM algorithms, where only the multiplications of complex numbers are considered. To be specific, if the number of TAs is 2 , the closed-form solution for the PRP can be found as shown in Section 3.3 , hence the corresponding complexity is

$$
C_{\mathrm{PRP}}=\left(2 N_{\mathrm{t}}^{2} N_{\mathrm{r}}-N_{\mathrm{t}} N_{\mathrm{r}}\right)+\frac{1}{2}\left(L^{2}-L\right)(2 L+8)
$$

Then, for the case of $N_{\mathrm{t}}>2$, the complexity of 'the proposed PRP Algorithm 1' and of 'the proposed PRP Algorithm 2' can be

\begin{tabular}{|c|c|c|c|c|}
\hline Adaptive algorithms & $\begin{array}{l}2 \times 2 \\
\text { OPSK }\end{array}$ & $\begin{array}{l}2 \times 2 \\
8 \mathrm{PSK}\end{array}$ & $\begin{array}{l}4 \times 2 \\
\text { QPSK }\end{array}$ & $\begin{array}{l}8 \times 2 \\
\text { QPSK }\end{array}$ \\
\hline ASM & 516 & 2172 & 2216 & 9168 \\
\hline $\begin{array}{l}\text { optimal max-FD PRP } \\
\text { algorithm }\end{array}$ & 108 & 684 & - & - \\
\hline proposed PRP Algorithm 1 & - & - & 522 & 1424 \\
\hline proposed PRP Algorithm 2 & - & - & 282 & 792 \\
\hline
\end{tabular}

Table 2 Complexity comparison of different adaptive algorithms for SM expressed, respectively, as (29) and (30)

$$
C_{\mathrm{PRP} 1}=\left(2 N_{\mathrm{t}}^{2} N_{\mathrm{r}}-N_{\mathrm{t}} N_{\mathrm{r}}\right)+N_{\mathrm{t}}+\left(\frac{N_{\mathrm{t}}^{2}-N_{\mathrm{t}}}{2}+N_{\mathrm{t}} N-N\right)(2 L-1)
$$

$$
\begin{aligned}
C_{\text {PRP2 }}= & \left(2 N_{\mathrm{t}}^{2} N_{\mathrm{r}}-N_{\mathrm{t}} N_{\mathrm{r}}\right)+N_{\mathrm{t}}+\frac{1}{2}\left(N_{\mathrm{t}}^{2}-N_{\mathrm{t}}+\left(L^{2}-L\right)\left(N_{\mathrm{t}}-1\right)\right) \\
& \times(2 L-1)+\frac{9}{2}\left(L^{2}-L\right)
\end{aligned}
$$

Moreover, the complexity of the ASM scheme of [18] is

$$
C_{\mathrm{ASM}}=\left(2 N_{\mathrm{t}}^{2} N_{\mathrm{r}}-N_{\mathrm{t}} N_{\mathrm{r}}\right)+9 N_{\mathrm{t}} L\left(N_{\mathrm{t}} L-1\right)
$$

Note that the complexity evaluations of the proposed PRP algorithms, as formulated in (28)-(30), are detailed in Appendix.

As shown in Table 2, the proposed PRP algorithms have a lower complexity than ASM. As regards to the feedback load, the PRP-aided SM-PSK scheme only has to feedback the TA index to be rotated and its corresponding phase-rotation value. Furthermore, the matched filter-based single-stream ML detector of [26, 27] can be adopted for the detection of our proposed PRP-aided SM-PSK, where the TA indices and the L-PSK symbols are detected separately.

\section{Simulation results}

In this section, we characterise the performance of both the conventional PSK-modulated SM scheme as well as of the corresponding max-FD-aided PRP schemes, namely that of the proposed optimal max-FD-based PRP assisted SM, of the PRP Algorithm 1 and of the PRP Algorithm 2. These performance results were also compared with that of the conventional PRP-aided SM schemes of [21], to the ASM arrangements of [18] and to the PA-aided SM schemes of [25] for transmission over independent Rayleigh block-flat MIMO channels [Note that the proposed PRP algorithm can be readily extended to the channels exhibiting time-domain correlation, where both the computational complexity and the feedback load are reduced, because only a single PRP matrix has to be calculated within each coherence interval.]. The reason we selected these schemes as our benchmarkers is because their design principle is the same as that of maximising the FD with the aid of a single-RF chain. Hence, these schemes have a similar overhead.

Fig. 4 shows the BER performance of the conventional PRP, as well as of the proposed optimal max-FD-aided PRP and of the optimal max-FD-based PA of [25]. In Fig. 4, the $(2 \times 1)$-element and $(2 \times 2)$-element MIMO channels using BPSK modulation are considered. For completeness, we added the theoretical upper bound of [11] for the conventional SM scheme. Moreover, for the $(2 \times 2)$-element MIMO channels, the performance curves of the conventional PRP-aided SM is not considered, because this method is only suitable for MISO channels. We observe in Fig. 4 that the PRP-aided SM schemes provide considerable signal-to-noise ratio (SNR) gains over the conventional SM schemes. Moreover, the proposed max-FD-assisted PRP outperforms the max-FD-aided PA algorithm of [25]. Furthermore, observed in Fig. 4 that according to Remark 1, we found that the conventional PRP and the proposed optimal max-FD-based arrangement achieve the same BER performance.

Fig. 5 compares the BER performances of the PRP-aided schemes and of the conventional ASM schemes for different PSK arrangements in $(2 \times 2)$ MIMO channels. As shown in Fig. 5, in the low-to-medium SNR regime, the proposed PRP-aided schemes outperform the ASM schemes, but they may perform worst than the ASM at high SNRs. This is due to the fact that these techniques exploit different properties of the MIMO channels, 
800

805

810

Fig. 5 BER comparison of the ASM and the proposed max-FD-based $P R P$-aided SM schemes in $(2 \times 2)$-element MIMO channels with different PSK schemes

815 when aiming for maximising the FD; hence, they may exhibit different BER advantages for different number of TAs. It is worth noting that different QAM schemes may be assigned to different TAs, relying on the near-instantaneous channel conditions in ASM; hence, the receiver has to detect these different constellations and hence imposes a higher complexity than the PSK-modulated PRP-aided SM. Moreover, as discussed in Section 2 and shown in Fig. 7, the proposed PRP scheme has a higher energy efficiency than the ASM scheme because of the constant-modulus property of the transmitted signals.

Moreover, Fig. 6 explores the attainable system performance relying on more antennas. To be specific, $(4 \times 2)$-element and $(8 \times 2)$-element MIMO channels are considered. Observed in Fig. 6 that the proposed max-FD-aided PRP-SM schemes achieve a $3-5 \mathrm{~dB}$ performance gain at $\mathrm{BER}=10^{-5}$ over the identical-throughput non-precoded $\mathrm{SM}$ scheme. Moreover, the low-complexity PRP Algorithm 2 achieves almost the same performance as the PRP Algorithm 1.

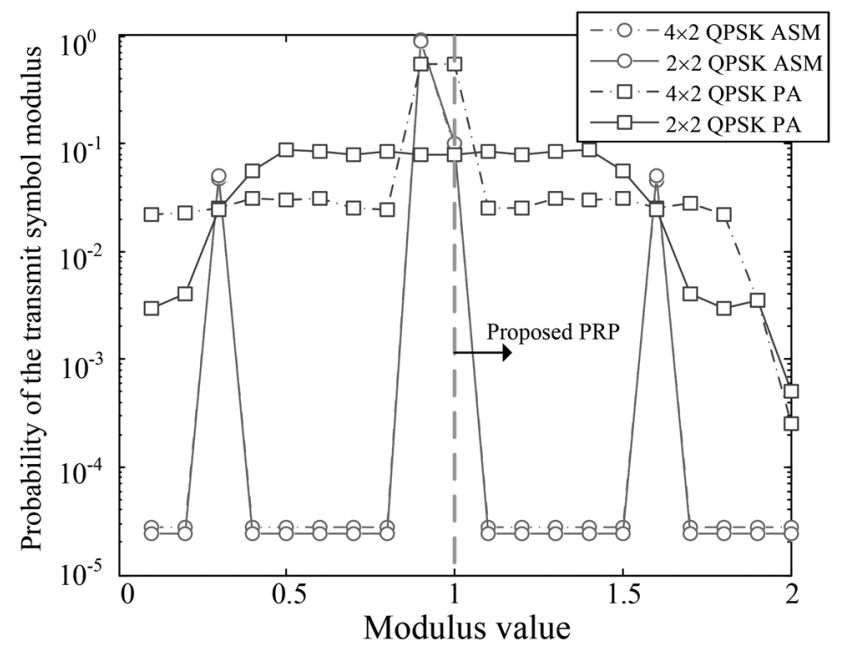

Fig. 7 Probability of the transmit symbol modulus for different ASM schemes

Furthermore, as shown in Figs. 4-6, the proposed PRP schemes are capable of achieving almost the same BER result as the identical-throughput ASM schemes, such as the $4 \times 2$ and $2 \times 2$ MIMO setups in Figs. 5 and 6. Moreover, as shown in Fig. 4, the proposed PRP schemes perform better than the PA-aided SM. In a word, the proposed PRP is an attractive alternative for ASM and PA-aided schemes in limited-feedback SM transmission in terms of BER. On the other hand, as shown in Fig. 7, in the proposed schemes the moduli of the transmit SM-MIMO signals are constant, whereas that of the ASM and the PA-aided SM schemes has a large variation. To be specific, the symbol-power within each RF chain of ASM and PA-aided SM is time-variant, depending on the PA matrix and ASM candidate invoked. This increases the realisation cost for practical communication systems. As shown in Fig. 7, this problem can be avoided by our proposed constant-modulus PRP-aided SM-PSK.
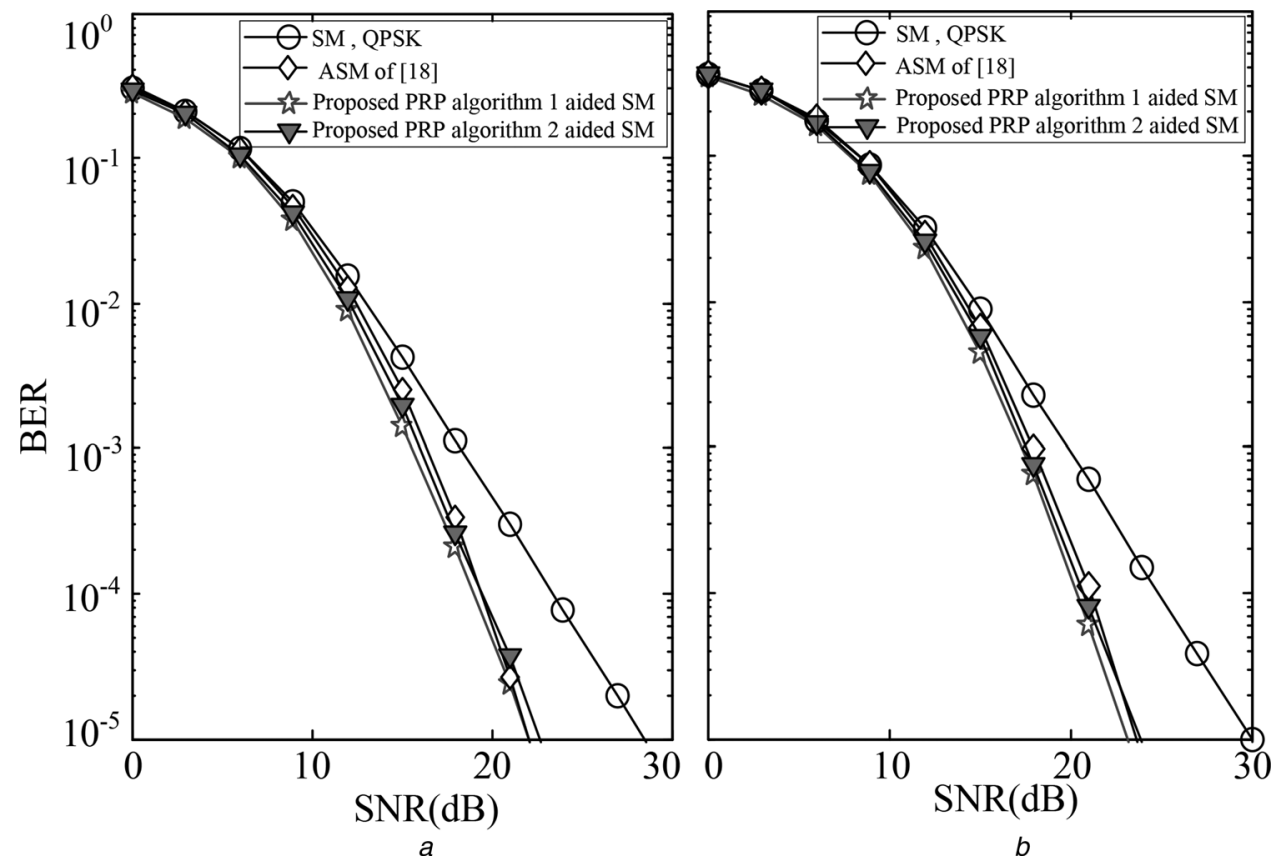

Fig. 6 BER comparison of various SM schemes in $(4 \times 2)$ - and $(8 \times 2)$-element MIMO channels

$a 4 \times 2$ MIMO

$b 8 \times 2$ MIMO 


\section{Conclusion}

In this paper, we have proposed a novel PRP-aided SM-PSK scheme for improving the achievable BER performance. Our PRP-aided SM scheme exhibits an attractive BER performance at a low complexity, despite its limited-feedback load. This system is also eminently suitable for single-RF-aided MIMO scenarios [28].

\section{Acknowledgments}

The financial support of the Doctor Foundation of Ministry of Education under the grant no. 20110185130003 and of the European Research Council's Advanced Fellow Grant is gratefully acknowledged.

\section{References}

1 Mesleh, R., Haas, H., Sinanovicí, S., et al.: 'Spatial modulation', IEEE Trans. Veh. Technol., 2008, 57, (4), pp. 2228-2241

2 Yang, Y., Jiao, B.: 'Information-guided channel-hopping for high data rate wireless communication', IEEE Commun. Lett., 2008, 12, (4), pp. 225-227

3 Jeganathan, J., Ghrayeb, A., Szczecinski, L., et al.: 'Space shift keying modulation for MIMO channels', IEEE Trans. Wirel Commun., 2009, 8, (7), pp. 3692-3703

4 Sugiura, S., Chen, S., Hanzo, L.: 'A universal space-time architecture for multiple-antenna aided systems', IEEE Commun. Surv. Tutor., 2012, 14, (2), pp. $401-420$

5 Sugiura, S., Chen, S., Hanzo, L.: 'Coherent and differential space time shift keying: a dispersion matrix approach', IEEE Trans. Commun., 2010, 58, (11), pp. $3219-3230$

6 Renzo, M.D., Haas, H., Ghrayeb, A., et al.: 'Spatial modulation for generalized MIMO: challenges, opportunities and implementation', Proc. IEEE, 2014, 102, (1), pp. 56-103

Chang, R.Y., Lin, S.J., Chung, W.H.: 'New space shift keying modulation with Hamming code-aided constellation design', IEEE Wirel. Commun. Lett., 2012, 1, (1), pp. 2-5

8 Başar, E., Aygölü, Ü., Panayırc1, E., et al.: 'Space-time block coded spatial modulation', IEEE Trans. Commun., 2011, 59, (3), pp. 823-832

9 Renzo, M.D., Haas, H., Grant, P.M.: 'Spatial modulation for multiple-antenna wireless systems: a survey', IEEE Commun. Mag., 2011, 49, (12), pp. 182-191

10 Hanzo, L., Ng, S.X., Keller, T., et al.: 'Quadrature amplitude modulation: from basics to adaptive trellis-coded, turbo-equalised and space-time coded OFDM, CDMA and MC-CDMA systems' (John Wiley and IEEE Press, 2004)

11 Renzo, M.D., Haas, H.: 'Bit error probability of spatial modulation (SM-) MIMO over generalized fading channels', IEEE Trans. Veh. Technol., 2012, 61, (3), pp. 1124-1144

12 Renzo, M.D., Haas, H.: 'Improving the performance of space shift keying (SSK) modulation via opportunistic power allocation', IEEE Commun. Lett., 2010, 14, (6), pp. 500-502

13 Ntontin, K., Renzo, M.D., Perez-Neira, A., et al.: 'Adaptive generalized space shift keying modulation', EURASIP J. Wirel. Commun. Netw., 2013

14 Xiao, Y., Tang, Q., Gong, L., et al.: 'Power scaling for spatial modulation with limited feedback', [Online]. Available at http://www.downloads.hindawi.com/ journals/ijap/aip/718482.pdf

15 Yang, D., Xu, C., Hanzo, L., et al.: 'Transmit-diversity-assisted space-shift keying for collocated and distributed/cooperative MIMO elements', IEEE Trans. Veh. Technol., 2011, 60, (6), pp. 2864-2869

16 Yang, P., Xiao, Y., Yu, Y., et al.: 'Adaptive spatial modulation for wireless MIMO transmission systems', IEEE Commun. Lett., 2011, 15, (6), pp. 602-605

17 Yang, P., Xiao, Y., Yu, Y., et al.: 'Link adaptation for spatial modulation with limited feedback', IEEE Trans. Veh. Technol., 2012, 61, (8), pp. 3808-3813

97518 Yang, P., Xiao, Y., Yu, Y., et al.: 'Simplified adaptive spatial modulation for limited-feedback MIMO', IEEE Trans. Veh. Technol., 2013, 62, (6), pp. 2656-2666

19 Maleki, M., Bahrami, H., Beygi, S., et al.: 'Space modulation with CSI: constellation design and performance evaluation', IEEE Trans. Veh. Technol., 2013, 62, (4), pp. 1623-1634

20 Maleki, M., Bahrami, H.R., Alizadeh, A., et al.: 'On the performance of spatial modulation: optimal constellation breakdown', IEEE Trans. Commun., 2014, 62, (1), pp. 144-157

21 Masouros, C.: 'Improving the diversity of spatial modulation in MISO channels by phase alignment', IEEE Commun. Lett., 2014, 18, (5), pp. 729-732

22 Goldsmith, A.: 'Wireless communication' (Cambridge University Press, New York, 2005), ch. 5

23 Collin, L., Berder, O., Rostaing, P., et al.: 'Optimal minimum distance-based precoder for MIMO spatial multiplexing systems', IEEE Trans. Signal Process., 2004, 52, (5), pp. 617-627

24 Vrigneau, B., Letessier, J., Rostaing, P., et al.: 'Optimal minimum distance-based precoder for MIMO spatial multiplexing systems', IEEE J. Sel. Top. Signal Process., 2008, 2, (2), pp. 135-146

25 Yang, P., Xiao, Y., Hanzo, L., et al.: 'Power allocation aided spatial modulation for ${ }_{990}$ Q10 limited-feedback MIMO systems', IEEE Trans. Veh. Technol., 2014, in press
$26 \mathrm{Xu}, \mathrm{C}$., Sugiura, S., Ng, S., et al.: 'Spatial modulation and space-time shift keying: optimal performance at a reduced detection complexity', IEEE Trans. Commun., 2013, 61, (1), pp. 206-216

27 Yang, P., Xiao, Y., Li, S.Q., et al.: 'An improved matched-filter based detection algorithm for space-time shift keying systems', IEEE Signal Process. Lett., 2012, 19, (5), pp. 271-274

28 Yang, P., Renzo, M.D., Hanzo, L., et al.: 'Design guidelines for spatial modulation', IEEE Commun. Surv. Tutor., 2014, in press

\section{Appendix}

The complexity expressions of the proposed PRP algorithms are presented by (28)-(30). This appendix provides more detailed information for this complexity assessment.

\subsection{Complexity of the optimal max-FD PRP algorithm using $N_{t}=2$}

Given channel matrix $\boldsymbol{H} \in \mathbb{C}^{N_{\mathrm{r}} \times N_{\mathrm{t}}}$ in Section 3.3, we first evaluated the elements of the matrix $\boldsymbol{H}^{\mathrm{H}} \boldsymbol{H}$ for the sake of determining the values of $\left|\boldsymbol{h}_{i}\right|^{2},\left|\boldsymbol{h}_{j}\right|^{2}$ and $\boldsymbol{h}_{i}^{\mathrm{H}} \boldsymbol{h}_{j}$ in (27). The associated complexity is given by

$$
\operatorname{comp}\left(\boldsymbol{H}^{\mathrm{H}} \boldsymbol{H}\right)=2 N_{\mathrm{t}}^{2} N_{\mathrm{r}}-N_{\mathrm{t}} N_{\mathrm{r}}
$$

where $\operatorname{comp}(\cdot)$ represents the number of complex-valued multiplications. Then, recall from Section 3.3 that the calculation of the $\left(\begin{array}{l}L \\ 2\end{array}\right)$ possible phase values in (20) imposes a complexity of

$$
\operatorname{comp}\left(\tan \theta_{1}\right)=\frac{9}{2}\left(L^{2}-L\right)
$$

Then, the FDs of all possible PRP matrices formed by (20) are computed by using (27) at a complexity of

$$
\operatorname{comp}\left(d_{\min }^{\text {all }}\right)=\left(\begin{array}{l}
L \\
2
\end{array}\right)(2 L-1)
$$

Finally, the total complexity of the proposed optimal max-FD PRP algorithm associated with $N_{\mathrm{t}}=2$ is given by (28), also taking into account (32)-(34).

\subsection{Complexity of the proposed PRP Algorithm 1 using $N_{t}>2$}

As shown in Section 3.4, we have to determine the elements of the matrix $\boldsymbol{H}^{\mathrm{H}} \boldsymbol{H}$. Then, the FD associated with the initial PRP matrix by using (27) is computed, which imposes a complexity of

$$
\operatorname{comp}\left(d_{\min }^{i n}\right)=\left(\begin{array}{c}
N_{\mathrm{t}} \\
2
\end{array}\right)(2 L-1)
$$

Note that the number of legitimate PRP matrices in (23) is $N_{\text {PRP1 }}=N$ and hence the FDs of these matrices are calculated by using (27) at a complexity of

$$
\operatorname{comp}\left(d_{\min }^{\mathrm{PRP} 1}\right)=N\left(N_{\mathrm{t}}-1\right)(2 L-1)
$$

Note that in (27) only the distances between the changed TA and other TAs has to be considered. To be specific, only one of the TAs assigns a new phase, whereas the other TAs may keep the phase values unchanged. Hence, there are $\left(N_{t}-1\right)$ possible TA pairs, which is the number of combinations of the changed TA and one of the unchanged TAs. For each pair, the complexity of evaluating the received distance is $(2 L-1)$. The final complexity result shown in (36). On the basis of (32), (35) and (36), the total complexity of the proposed PRP Algorithm 1 is formulated in (29). 
9.3 Complexity of the proposed PRP Algorithm 2 using $N_{t}>2$

1060

By contrast, for the proposed PRP Algorithm 2 of Section 3.5, the legitimate PRP matrices are based on using (20) rather than (23), which imposes a complexity of comp $\left(\tan \theta_{1}\right)$. Moreover, since there are $N_{\mathrm{PRP} 2}=\left(\begin{array}{l}L \\ 2\end{array}\right)$ possible PRP solutions, compared with 1065

(36), the complexity of computing the FDs of $N_{\mathrm{PRP} 2}$ PRP matrices is given by

$$
\operatorname{comp}\left(d_{\min }^{\mathrm{PRP} 2}\right)=N_{\mathrm{PRP} 2}\left(N_{\mathrm{t}}-1\right)(2 L-1)
$$

Hence, based on (32), (33), (35) and (37), we arrive at the complexity of the proposed PRP Algorithm 2 as in (30). 
Author Queries

Ping Yang, Yue Xiao, Bo Zhang, Mohammed El-Hajjar, Shaoqian Li, Lajos Hanzo

Q1 Author "El-Hajjar" is not linked to any of the affiliations. Please check and provide the link.

Q2 Please check and confirm the email id of the corresponding author.

Q3 The sentence [The conventional open-loop SM schemes [11] only offer receive diversity gains.] has been changed to [The conventional open-loop SM schemes [11] only offer received diversity gains.]. Please confirm that this is what you intended, otherwise amend the sentence to convey your exact meaning.

Q4 Please check and confirm the insertion of subcaption of Figs. 1 and 6.

IET style for matrices and vectors is to use bold italics. Please check that we have identified all instances.

Q6 Please define the acronym PEP.

Q7 Please provide the publisher location in Ref. [10].

Q8 Please provide volume and page range for Ref. [13].

Q10 Please update Refs. [25, 28]. 